\title{
The Effect of Multidomain Lifestyle Intervention on Daily Functioning in Older People
}

\author{
Jenni Kulmala, PhD, *广* Tiia Ngandu, MD, PhD, *广 Satu Havulinna, PhD, ${ }^{\S}$ Esko Levälabti, MSc, * \\ Jenni Lehtisalo, MSc, * Alina Solomon, MD, PhD, ${ }^{\dagger / l}$ Riitta Antikainen, MD, PhD, ${ }^{\| * * \dagger \dagger}$ \\ Tiina Laatikainen, MD, PhD, **\$s Pauliina Pippola, MD, ${ }^{\text {III }}$ Markku Peltonen, PhD,* \\ Rainer Rauramaa, MD, PhD, "III Hilkka Soininen, MD, PhD, ${ }^{\mathscr{T} * * *}$ Timo Strandberg, MD, PhD, ${ }^{\| \dagger+\dagger}$

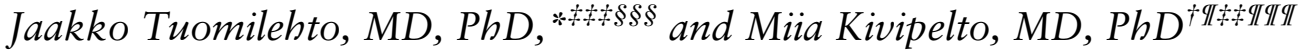

OBJECTIVE: To investigate the effect of a 2-year multidomain lifestyle intervention on daily functioning of older people.

DESIGN: A 2-year randomized controlled trial (ClinicalTrials. gov, NCT01041989).

SETTING: Finnish Geriatric Intervention Study to Prevent Cognitive Impairment and Disability.

PARTICIPANTS: A total of 1260 older adults, with a mean age of 69 years at the baseline, who were at risk of cognitive decline.

INTERVENTION: A multidomain intervention, including simultaneous physical activity intervention, nutritional counseling, vascular risk monitoring and management, and cognitive training and social activity.

From the *Public Health Promotion Unit, National Institute for Health and Welfare, Helsinki, Finland; 'Division of Clinical Geriatrics, Center for Alzheimer Research, Care Sciences and Society (NVS), Karolinska Institutet, Stockholm, Sweden; ${ }^{*}$ School of Health Care and Social Work, Seinäjoki University of Applied Sciences, Seinäjoki, Finland; ${ }^{\S}$ Ageing, Disability and Functioning Unit, National Institute for Health and Welfare, Helsinki, Finland; "Institute of Clinical Medicine/Neurology, University of Eastern Finland, Kuopio, Finland; "Center for Life Course Health Research, University of Oulu, Oulu, Finland; * Medical Research Center Oulu, Oulu University Hospital, Oulu, Finland; ${ }^{\dagger \dagger}$ Oulu City Hospital, Oulu, Finland; ${ }^{*}$ Institute of Public Health and Clinical Nutrition, University of Eastern Finland, Kuopio, Finland;

$\$$ Joint Municipal Authority for North Karelia Social and Health Services (Siun Sote), Joensuu, Finland; "IIISeinäjoki Health Centre, Seinäjoki, Finland;

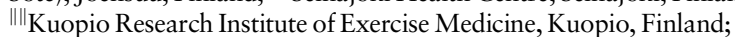

$* *$ Neurocenter, Department of Neurology, Kuopio University Hospital, Kuopio, Finland; University of Helsinki and Helsinki University Hospital, Helsinki, Finland; ${ }^{+*}$ Department of Public Health, University of Helsinki, Helsinki, Finland; ${ }^{\S \S}$ National School of Public Health, Madrid, Spain; and the

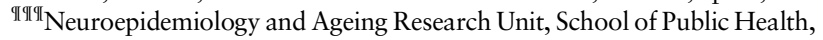
Imperial College London, London, United Kingdom.

Address correspondence to Jenni Kulmala, PhD, Chronic Disease Prevention Unit, National Institute for Health and Welfare, P.O. Box 30 00271, Helsinki, Finland. E-mail: jenni.kulmala@thl.fi

DOI: $10.1111 /$ jgs. 15837
MEASUREMENTS: The ability to perform daily activities (activities of daily living [ADLs] and instrumental ADLs) and physical performance (Short Physical Performance Battery).

RESULTS: The mean baseline ADL score was $18.1(\mathrm{SD}=2.6)$ points; the scale ranges from 17 (no difficulties) to 85 (total ADL dependence). During the 2-year intervention, the ADL disability score slightly increased in the control group, while in the intervention group, it remained relatively stable. Based on the latent growth curve model, the difference in the change between the intervention and control groups was -0.95 (95\% confidence interval $[\mathrm{CI}]=-1.61$ to -0.28$)$ after 1 year and -1.20 $(95 \% \mathrm{CI}=-2.02$ to -0.38$)$ after 2 years. In terms of physical performance, the intervention group had a slightly higher probability of improvement (from score 3 to score 4; $P=.041$ ) and a lower probability of decline (from score 3 to scores $0-2$; $P=.043)$ for chair rise compared to the control group.

CONCLUSION: A 2-year lifestyle intervention was able to maintain the daily functioning of the at-risk older population. The clinical significance of these results in this fairly well-functioning population remains uncertain, but the study results hold promise that healthy eating, exercise, and cognitive and social activity may have favorable effects on functional independence in older people. J Am Geriatr Soc 00:1-7, 2019.

Key words: clinical trial; disablement process; functional performance; preventative healthcare

A dequate ability to perform daily tasks and physical performance are prerequisites for independent living. Disability refers to difficulties performing or the inability to perform basic activities of daily living (BADLs), such as bathing, 
dressing, and transferring into a bed, ${ }^{1}$ or more demanding instrumental activities of daily living (IADLs), such as coping with housework, handling finances, or using a telephone. ${ }^{2}$ The disablement model by Verbrugge and Jette (1994) demonstrates how functional limitations usually precede disability in BADLs and IADLs. ${ }^{3}$ Functional decline and disability increase the risk of several adverse health events, including injuries, ${ }^{4}$ hospital care, and premature death. ${ }^{5}$

Lifestyle-related risk factors for disability have been relatively widely reported. Longitudinal studies have reported that physically inactive persons reach the disability threshold level earlier. ${ }^{6} \mathrm{~A}$ healthy diet and avoiding obesity are also associated with lower risk of functional decline in older people, ${ }^{7}$ and several chronic conditions, including high blood pressure and cardiovascular diseases, may accelerate the decline. ${ }^{8}$ However, only a few large randomized controlled trials (RCTs) have investigated whether simultaneous changes in diet, increasing physical exercise, or modifying cardiovascular risks slow down the progression or prevent BADL/IADL disabilities. A few recent intervention studies with different designs have not produced uniform results. ${ }^{9,10}$

This study reports findings from the Finnish Geriatric Intervention Study to Prevent Cognitive Impairment and Disability (FINGER), which is the first large RCT targeting persons at risk of cognitive decline with a multidomain intervention, including simultaneous physical activity, nutritional counseling, vascular risk monitoring and management, and cognitive training and social activity. ${ }^{11,12}$ The FINGER study has demonstrated significant intervention effects on the primary outcome (overall cognition) and main cognitive secondary outcomes (executive functioning and processing speed). Most secondary outcomes ${ }^{12}$ of the FINGER study have already been reported. ${ }^{13-15}$ For health resources, biomarkers and mortality analyses are ongoing. In the current study, we evaluated whether a multidomain lifestyle intervention reduces a decline in physical performance measured by testing standing balance, a timed sit to stand, and $4 \mathrm{~m}$ comfortable walking time. ${ }^{12} \mathrm{We}$ also conducted exploratory analyses investigating the intervention effects on disability measured with a self-reported assessment of the ability to perform daily living activities.

\section{METHODS}

\section{Participants}

FINGER (ClinicalTrials.gov identifier: NCT01041989) ${ }^{11-13}$ includes altogether 1260 independently living older persons from six cities in Finland. The full FINGER study protocol, recruitment of the participants, baseline characteristics, and outcomes have been reported in detail previously. ${ }^{11,12}$ The inclusion criteria were that the participants were at an age of 60 to 77 years at the start of the study, had a Cardiovascular Risk Factors, Aging and Dementia Risk Score ${ }^{16}$ of 6 points or higher, and had a cognitive performance at the mean level or slightly lower than expected for their age, according to Finnish population norms tested with the Consortium to Establish a Registry for Alzheimer's Disease neuropsychological battery. ${ }^{17}$ Persons with previously diagnosed or suspected dementia, disorders affecting safe engagement in the intervention (eg, malignant disease, major depression, symptomatic cardiovascular disease, revascularization within 1 year previously), severe loss of vision, hearing, or communicative ability, and disorders preventing cooperation, as judged by the study physician, were excluded.

\section{FINGER Intervention}

Participants were randomly assigned into groups receiving an intensive multidomain intervention or regular health advice (control group) at a 1:1 ratio. Computer-generated allocation was carried out in blocks of four (two individuals randomly allocated to each group) at each site after the baseline by the study nurse. The group allocation was not actively explained to the participants. The intervention components have been described in detail previously. ${ }^{11,12}$ Briefly, the multidomain intervention included simultaneous physical activity intervention, nutritional counseling, vascular risk monitoring and management, and cognitive training and social activity. The nutritional component was based on the Finnish Nutrition Recommendations ${ }^{18}$ and was conducted by study nutritionists (three individual sessions and seven to nine group sessions). The physical activity component was based on international guidelines ${ }^{19}$ and a modified version of the Dose Responses to Exercise Training study protocol. ${ }^{20}$ Training was guided by physiotherapists at a gym and consisted of individually tailored programs for progressive muscle strength training (one to three times per week) and aerobic exercise (two to five times per week). Exercises improving postural balance were also included. Cognitive training included 10 group sessions led by a psychologist. Individual sessions consisted of independent computer-based training at home or at the study site. Two 6-month periods included 72 training sessions each (three times per week, 10-15 minutes per session). The training program was a web-based in-house developed computer program, which included several cognitive tasks adapted from previous RCTs. ${ }^{21}$ Social activities were stimulated through the numerous group meetings. The management of metabolic and vascular risk factors was based on national evidence-based guidelines. ${ }^{22-24}$ Blood pressure, weight, body mass index (BMI), and hip and waist circumference were regularly examined, and the participants received advice on leading a healthy lifestyle. Adherence was the highest for cardiovascular monitoring and individual nutritional counseling, intermediate for cognitive training and nutrition group visits, and lower for independent gym training and computerized computer training (more detailed adherence data are presented in Table S1).

The FINGER study was approved by the coordinating ethics committee of the Hospital District of Helsinki and Uusimaa. The participants gave their informed written consent prior to the study.

\section{Outcome Measures}

\section{Activities of Daily Living Disability}

BADLs and IADLs were assessed using questionnaires. ${ }^{1,2,25}$ Hereafter in the text, we use the term ADL to refer to the questionnaire covering both BADL and IADL components. The questionnaire included 17 items. The ability to perform the following daily activities was assessed: toileting, eating, bathing, moving to and out of bed, dressing, moving indoors, walking up and down stairs, cutting toe nails, taking and handling medications, using a telephone, cooking, light housework, handling finances, doing laundry, using public transportation, shopping, 
and heavy housework. The response options were (1) able to independently perform the activity without any difficulties; (2) able to independently perform the activity, but with minor difficulties; (3) able to independently perform the activity, but with major difficulties; (4) able to perform the activity only when assisted; and (5) not able to perform the activity even when assisted. A sum score based on the ADL questions (range = 17-85), at the baseline, 12-month follow-up, and 24-month follow-up, was calculated. Higher score indicated poorer daily functioning, and a score of 17 indicated no problems in any task.

\section{Physical Performance}

The Short Physical Performance Battery (SPPB) ${ }^{26}$ was administered to all participants before and after the intervention period. The SPPB consists of three subtests: a hierarchical test of balance, a 4-m walk at a normal pace, and standing up from a chair five times consecutively. In the balance test, the participants were asked to remain standing with their feet as close together as possible, then in a semitandem position (ie, with the heel of one foot alongside the big toe of the other foot), and finally in a tandem position (ie, with the heel of one foot directly in front of the other foot and touching it). Each position had to be held for 10 seconds. For gait speed, the time required to walk $4 \mathrm{~m}$ at a normal pace was measured. This test was repeated twice, and the better time of the two was used in the analyses. For the chair rise test, participants were asked to stand up and sit down in a chair five times as quickly as they could with their arms crossed over the chest, and the time required was measured. Each test was scored from 0 (worst performance) to 4 (best performance). We have previously reported analyses with the continuous SPPB sum score as an outcome. The intervention and control groups did not differ significantly, but the estimate $(95 \%$ confidence interval $[\mathrm{CI}])$ was $0.03(-0.04$ to 0.10$)$ in favor of intervention ${ }^{13}$ The present analyses were done to investigate the intervention effects on the chair rise, balance, and walking ability, because the intervention may have different effects on these tasks, which vary in difficulty.

\section{Statistical Analyses}

We applied a latent growth curve modeling approach for the ADL using all three time points (baseline, 12 months, and 24 months) to analyze the change during 24 months. To account for a nonnormal distribution of the ADL, a censored normal model was assumed with a minimum ADL score (17) as censoring point (ie, floor effect). Change was assumed to be nonlinear by estimating the shape parameter (ie, factor loading for a 12-month score) of the growth factor as free as opposed to a linear change model where the shape parameter is fixed. Mplus version 5.1 was used with full information maximum likelihood (FIML) estimation. FIML uses all available data by assuming that missing data are random. Because of the censored normal model, the integration algorithm was applied.

For ordinal scale SPPB scores, an ordered logistic regression model was applied. For analyses, participants with scores of 0 to 2 in each domain were combined due to the limited number of persons in these categories. A 24-month score was the model outcome, while the baseline score, group, and baseline score-group interaction were the covariates. Based on an ordered logistic regression model, conditional transitions by model covariate categories were estimated with $95 \%$ CIs and differences between groups were tested. An omnibus $P$ value for all transitions was calculated by testing the joint hypotheses (Stata's test command, applying accumulate option).

Additional analyses using logistic regression, including only the persons without any difficulties in the ADLs (total score $=17, \mathrm{n}=774$ ) at the baseline and persons without any difficulties in the SPPB (total score $=12, \mathrm{n}=460$ ) at the baseline, were conducted to investigate the risk for ADL and SPPB incident difficulties in the intervention and control groups.

\section{RESULTS}

\section{Population Characteristics}

At the baseline, the participants' ages ranged from 60 to 77 years (mean $=69$ years). The mean Mini-Mental State Examination score at the baseline was 26.7 points, the mean BMI was $28.2 \mathrm{~kg} / \mathrm{m}^{2}$, and the mean education level was 10 years. A total of $71 \%$ were physically active at least twice a week. The baseline characteristics for the participants in the intervention and control groups are presented in Table 1. The flowchart shows the number of participants assessed for eligibility and the flow of participants (Figure 1).

The ADL sum score in FINGER participants ranged from 17 to 46 at the baseline, from 17 to 51 at the 12-month follow-up, and from 17 to 59 at the 24-month follow-up. In comparison, the full range of the ADL scale was from 17 (no difficulties) to 85 (total ADL dependence). The mean ADL total score at the baseline was 18.1 ( $\mathrm{SD}=$ 2.6) points, and the mean SPPB score was 10.8 (12 points indicating no difficulties). Table 2 shows the mean ADL and SPPB scores at the baseline and the mean change over time. The number of persons with difficulties in ADL and functional performance are presented in Table S2.

Results from the latent growth curve modeling showed that the ADL disability score slightly increased in the control group (the 12-month estimated mean change was 0.88 [95\% $\mathrm{CI}=0.34-1.42]$; and for 24 months, it was $1.10[95 \% \mathrm{CI}=$ $0.53-1.67])$, while in the intervention group, it remained stable (the 12-month estimated mean change was $-0.12[95 \%$ $\mathrm{CI}=-0.61$ to 0.38$]$; and for 24 months, it was $-0.15[95 \%$ $\mathrm{CI}=-0.77$ to 0.48$]$ ). The difference in the change between the intervention and control groups was $-0.95(95 \% \mathrm{CI}=$ -1.61 to -0.28$)$ after 1 year and $-1.20(95 \% \mathrm{CI}=-2.02$ to -0.38 ) after 2 years, indicating increased disability in the control group compared to the intervention group (Figure 2). The effect size (Cohen's d) and the 95\% CI for ADL was $-0.31(95 \% \mathrm{CI}=-0.53$ to -0.09$)$ after 1 year and -0.39 $(95 \% \mathrm{CI}=-0.66$ to -0.12$)$ after 2 years.

The FINGER participants displayed good physical performance, and it remained relatively stable over 2 years. Distributions of the SPPB scores at baseline and at the 2-year follow-up are shown in Table S3. A total of 227 participants improved $(30 \%)$ and 308 declined $(22 \%)$ in their total SPPB scores over the 2 years, with a mean improvement of $1.5(\mathrm{SD}=0.9)$ points and a mean decline of -1.2 $(\mathrm{SD}=0.5)$ points. Significant differences in the transitions 
Table 1. FINGER Study Population in the Intervention and Control Groups

\begin{tabular}{|c|c|c|c|}
\hline Characteristics & $\begin{array}{l}\text { Participants } \\
\text { With } \\
\text { Information } \\
\text { Available }\end{array}$ & $\begin{array}{l}\text { Intervention } \\
\text { Group }^{\mathrm{a}}\end{array}$ & $\begin{array}{l}\text { Control } \\
\text { Group }^{\mathrm{a}}\end{array}$ \\
\hline Sex (women) & 1260 & $286(45)$ & $303(48)$ \\
\hline Age, y & 1260 & $69.7(4.6)$ & $69.4(4.7)$ \\
\hline Education, y & 1258 & $10.0(3.5)$ & $10.0(3.4)$ \\
\hline $\begin{array}{l}\text { Physical activity at least } \\
\text { twice a week }\end{array}$ & 1247 & $436(70)$ & $447(72)$ \\
\hline Body mass index, $\mathrm{kg} / \mathrm{m}^{2}$ & 1249 & $28.3(4.5)$ & $28.1(4.9)$ \\
\hline Diabetes & 1253 & $86(14)$ & 79 (13) \\
\hline MMSE score ${ }^{b}$ & 1257 & $26.7(2.1)$ & $26.8(2.0)$ \\
\hline ADL score (baseline) ${ }^{\mathrm{C}}$ & 1210 & $18.2(2.9)$ & $18.1(2.4)$ \\
\hline SPPB score (baseline) ${ }^{d}$ & 1210 & $10.8(1.4)$ & $10.8(1.4)$ \\
\hline
\end{tabular}

${ }^{\mathrm{a}}$ Data are given as number (percentage) or mean (SD).

${ }^{\mathrm{b}}$ The MMSE score range is from 0 to 30 (a higher score indicates better cognition).

${ }^{\mathrm{c}}$ The ADL score range is from 17 to 85 (a higher score indicates more disability).

${ }^{\mathrm{d}}$ The SPPB score range is from 0 to 12 (a higher score indicates better physical performance).

Abbreviations: ADL, activity of daily living; FINGER, Finnish Geriatric Intervention Study to Prevent Cognitive Impairment and Disability; MMSE, Mini-Mental State Examination; SPPB, Short Physical Performance Battery.

between the groups were observed only for the chair rise test, where the intervention group had a slightly higher probability of improving and a lower probability of decline compared to the control group. All the transitions in the chair rise, balance, and walking test, according to the baseline score and group, and differences between the intervention and control group (percentage with 95\% CIs) are shown in Tables $\mathrm{S} 4$ to $\mathrm{S} 6$.

The risk of incident ADL disability among those without any baseline difficulties during the 2-year intervention period was significantly lower in the intervention group (odds ratio $[\mathrm{OR}]=0.57 ; 95 \% \mathrm{CI}=0.39-0.84$ ). Intervention was not associated with incident limitations in physical performance $(\mathrm{OR}=1.29 ; 95 \% \mathrm{CI}=0.84-2.00)$.

\section{DISCUSSION}

This study showed that a 2-year multidomain intervention could maintain daily functioning of older people who are at risk of cognitive decline. The ADL disability score slightly increased in the control group during the intervention, while in the intervention group, it remained stable. Intervention also had small favorable effects on chair rise. The clinical significance of these findings observed in a fairly well-functioning population remains uncertain. Although statistically significant, the observed changes in ADL score were small during the short within-study follow-up period of 2 years. The trial was powered to demonstrate the effectiveness of the intervention, and the true potential of the intervention will become clear after a longer follow-up time. Still, this beneficial effect on ADLs after 2 years in an older population who are at risk for cognitive decline may be relevant for public health.

The current study is among the first trials showing the effects of a multifactorial lifestyle intervention on the daily functioning and physical performance of community-dwelling older people who are at risk of cognitive decline. Maintaining or improving physical functioning and detecting the first signs

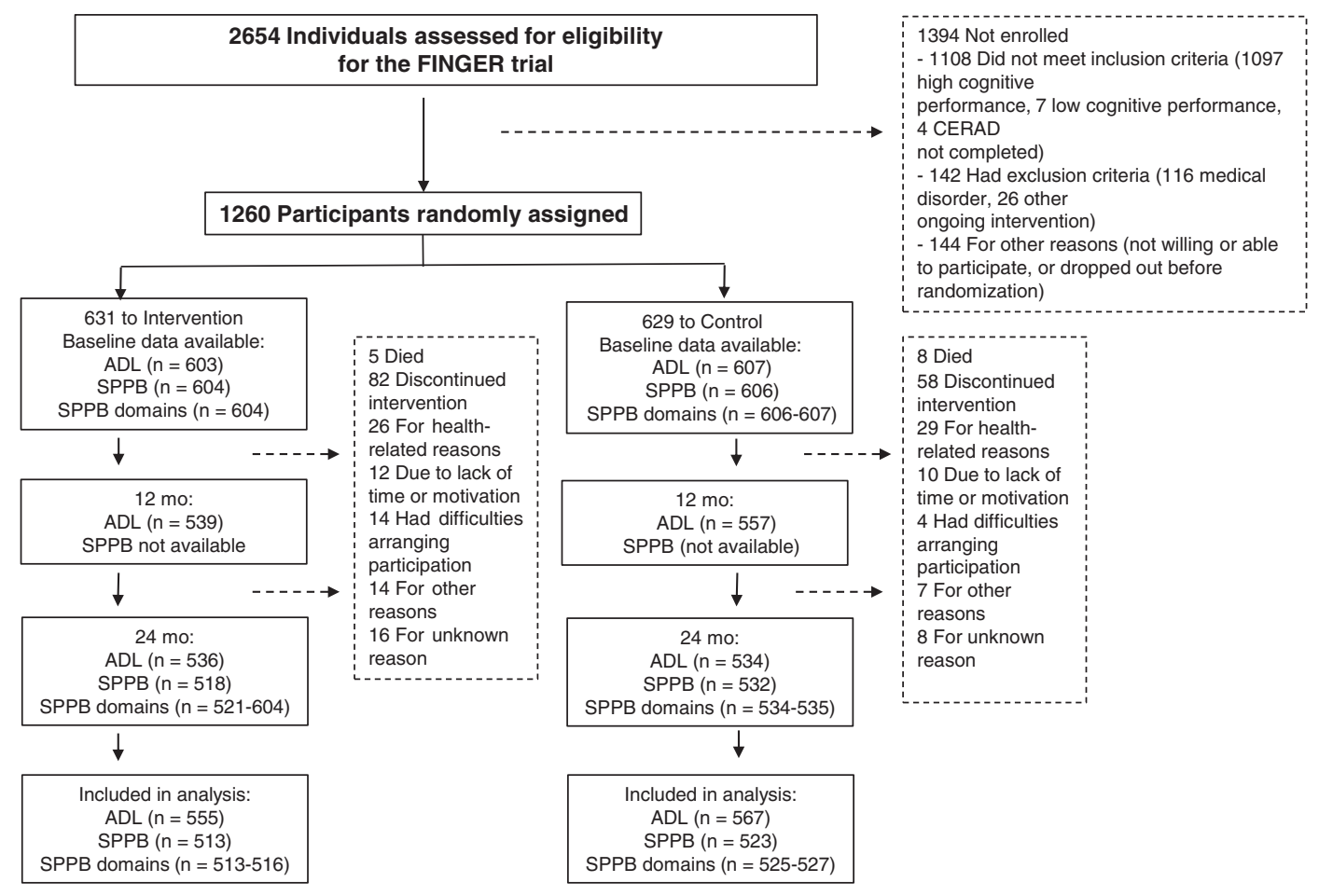

Figure 1. Study flowchart. ADL, activity of daily living; CERAD, Consortium to Establish a Registry for Alzheimer's Disease; FINGER, Finnish Geriatric Intervention Study to Prevent Cognitive Impairment and Disability; SPPB, Short Physical Performance Battery. 
Table 2. The Mean ADL and SPPB Scores at Baseline and Mean Change Over Time

\begin{tabular}{|c|c|c|c|}
\hline Variable & Baseline & $12 \mathrm{mo}$ & $24 \mathrm{mo}$ \\
\hline \multicolumn{4}{|c|}{ ADL Factors } \\
\hline \multicolumn{4}{|c|}{ ADL disability score at baseline and change, mean (SD) ${ }^{\mathrm{a}}$} \\
\hline All $(n=1210)$ & $18.1(2.6)$ & $0.4(2.0)$ & $0.5(2.1)$ \\
\hline Intervention group $(n=603)$ & $18.2(2.9)$ & $0.3(2.2)$ & $0.5(2.2)$ \\
\hline Control group $(n=607)$ & $18.1(2.4)$ & $0.5(1.8)$ & $0.5(2.0)$ \\
\hline \multicolumn{4}{|c|}{ Participants who changed from no disability to at least some disability during $24 \mathrm{mo} \%{ }^{\mathrm{b}}$} \\
\hline Intervention group & & 17 & 16 \\
\hline Control group & & 23 & 25 \\
\hline \multicolumn{4}{|c|}{ Physical Performance Factor } \\
\hline \multicolumn{4}{|c|}{ SPPB score at baseline and change, mean (SD) ${ }^{c}$} \\
\hline All $(n=1210)$ & $10.8(1.4)$ & NA & $-0.2(1.1)$ \\
\hline Intervention group $(n=604)$ & $10.8(1.4)$ & NA & $-0.2(1.1)$ \\
\hline Control group $(n=606)$ & $10.8(1.4)$ & NA & $-0.2(1.1)$ \\
\hline
\end{tabular}

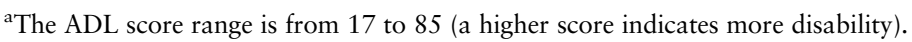

${ }^{\mathrm{b}} \mathrm{A}$ score of 18 or more refers to at least some difficulties.

${ }^{\text {c}}$ The SPPB score range is from 0 to 12 (a higher score indicates better performance).

Abbreviations: ADL, activity of daily living; NA, Not available; SPPB, Short Physical Performance Battery.

of functional decline at the ages of 65 to 80 years may have farreaching implications for improving functional independence after the age of 80 years. ${ }^{27}$ In most countries, the oldest old are now the fastest-growing part of the overall population, and it is essential to find ways to promote their healthy and independent living. The multidomain intervention was associated with lower odds of incident ADL disabilities. This may have clinical significance, since it has been previously shown that moving from being robust to physically frail within a few-years' time significantly increases the mortality risk. ${ }^{28}$ Our findings are supported by the cohort study showing that better walking ability and a better-quality diet were significantly associated with a compression of the disabled period. ${ }^{29}$

In our study of a population with a mean age of 69 years, 36\% had at least some difficulties in daily activities at the baseline and $62 \%$ had at least some difficulties in

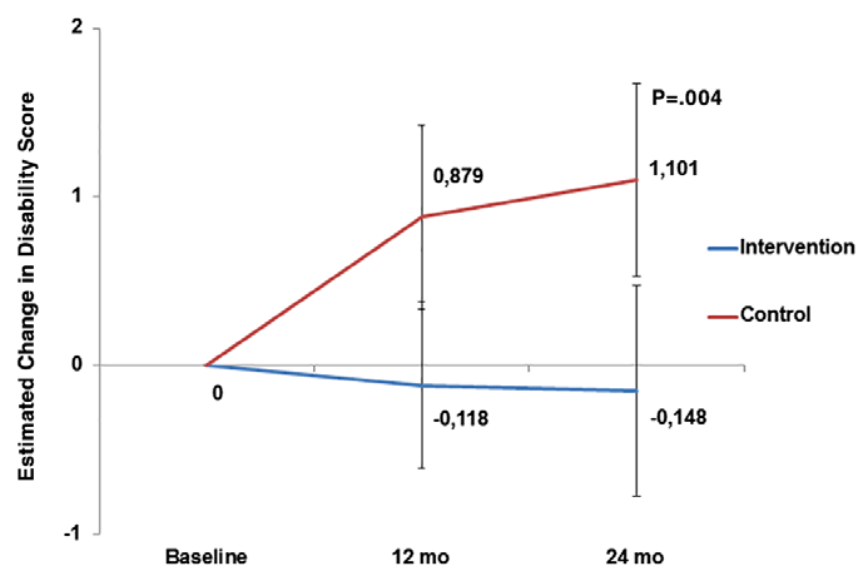

Figure 2. Change in the activity of daily living (ADL) score during the 2-year intervention period. The figure shows the estimated mean change in the ADL score (range = 17-85) from the baseline up to 12 and 24 months (a higher score suggests greater disability). The error bars are confidence intervals. A latent growth curve modeling approach using all three time points (baseline, 12 months, and 24 months) was used. The $P$ value is from the Wald Test of Parameter Constraints. physical performance. However, the mean ADL total score at the baseline was 18 points (17 points indicating no difficulties in ADLs) and the mean SPPB score was 11 points (12 points indicating no difficulties), which indicated that the participants in the FINGER study were healthy and functionally independent. Previous studies have shown that the ADL/IADL disability ranges between $2 \%$ and $20 \%^{30-32}$ among older people aged 60 to 80 years, depending on the age group and ADL disability definition used. Thus, our sample can be considered representative of the population at this age. Changes in outcomes during the 2-year intervention in this well-functioning population were relatively small. In single-domain intervention studies with similar outcomes, larger changes have been observed; however, the study populations have been older and with more functional limitations at the baseline. ${ }^{33,34}$

A few study limitations must be considered. Due to a relatively well-functioning study population, there was only a little room for improvement in physical functioning during the 2-year period and the outcome measures used may have had a ceiling effect. The currently ongoing FINGER 7-year follow-up phase will provide more information on the longer-term effects of the intervention as the individuals get older and most likely develop more impairments. The aim of the FINGER intervention was to target an at-risk population and to start early before cognitive or functional decline has started. Therefore, it is not easy to see the intervention effects after 2 years. However, by starting early, we will most likely achieve better long-term effects. Also due to the small number of participants in some of the categories in the functional performance tests, the lack of statistical power in some of the analyses may limit the findings. Although the subgroup analyses showed that the intervention prevented incident ADL limitations, these results should be interpreted with caution because the changes were small, and the two groups were slightly different at the baseline and adjusting for the baseline level may not correct this completely. The main outcome of the FINGER study was cognition, and the multidomain intervention was not specifically planned for improving daily functioning. 
Due to the multidomain approach, it is not possible to exactly state the individual effects of different intervention components on the observed effects. For similar outcomes, previous studies with physical activity interventions have provided beneficial effects on physical functioning ${ }^{35,36}$ and, therefore, it is likely that intensive physical activity training is one of the key components of the FINGER program. In future studies, the FINGER-type multimodal intervention model needs to be investigated further, particularly with regard to the contribution of each component to the overall effect.

The strengths of this study include the controlled randomized study design with a large sample size, longer duration than in most trials, detailed outcome assessments, and high-quality data collection. The multidomain lifestyle intervention was found to be feasible and safe, with no serious adverse events. ${ }^{13}$ Also, the dropout rate was low (12\%). We used validated performance-based outcome measures of physical performance and the widely used scale of ADL functions. This study provides a reference frame for changes in daily functioning for older people who are at risk of dementia but have not yet developed significant impairments.

Independent daily functioning and physical performance in older people are influenced by a wide range of health- and lifestyle-related factors. A multidomain intervention simultaneously targeting multiple risk factors may prevent ADL disabilities in the older at-risk population. The FINGER study has shown that modifying older at-risk people's lifestyles has beneficial effects on cognition ${ }^{13}$ quality of life, ${ }^{14}$ and, as shown in the present study, daily functioning. This study, together with future multidomain intervention studies following the protocol of the FINGER study, will provide additional understanding on the benefits of multidomain lifestyle interventions on the prevention of disability among old people at risk of cognitive decline.

\section{ACKNOWLEDGEMENTS}

We thank all members of the Finnish Geriatric Intervention Study to Prevent Cognitive Impairment and Disability study group for their cooperation in data collection and management. We are indebted to all the participants for their commitment to the study.

Financial Disclosure: The Finnish Geriatric Intervention Study to Prevent Cognitive Impairment and Disability is financially supported by Academy of Finland's Responding to Public Health Challenges Research Programme; Academy of Finland grants 278,457 and 317,465 (M.K.) and 287,490, 294,061, and 319,318 (A.S.); Academy of Finland Key Project Funding 305,810; European Research Council 804,371 (A.S.); La Carita Foundation; Juho Vainio Foundation; Finnish Medical Foundation (T.N.); Finnish Social Insurance Institution; Ministry of Education and Culture Research Grant (M.K., J.K.); EVO/VTR grants of University Hospitals of Kuopio, Oulu, and Turku; Seinäjoki Central Hospital and Oulu City Hospital; Yrjö Jahnsson Foundation; Finnish Cultural Foundation; South Ostrobothnia Regional Fund; Swedish Research Council; Alzheimerfonden; Stockholm County Council; Alzheimer's Research and Prevention Foundation USA; AXA Research Fund; Knut and Alice Wallenberg Foundation Sweden; Center for Innovative Medicine at Karolinska Institutet Sweden; Stiftelsen Stockholms Sjukhem Sweden; and Konung Gustaf V:s och Drottning Victorias Frimurarstiftelse Sweden.
Conflict of Interest: Kulmala, Havulinna, Ngandu, Levälahti, Lehtisalo, Peltonen, Pippola, and Solomon report no disclosures. Kivipelto has served on scientific advisory boards for Pfizer Inc and Elan Corporation; has served on the editorial advisory board of the Journal of Alzheimer's Disease; and has received speaker honoraria from Janssen, Novartis, and Pfizer Inc. Strandberg has made various cooperative agreements (research, consultative, educational) with several companies marketing cardiovascular drugs. Soininen has served on advisory boards of ACImmine and Merck.

Author Contributions: T.N., A.S., R.A., T.L., M.P., R.R., T.S., J.T., H.S., and M.K. conceived and designed the trial. T.N., J.L., and M.K. coordinated the trial. J.L. (nutrition), R.R. and S.H. (physical exercise), T.N. (cognitive training), and T.S., R.A., and J.T. (vascular risk monitoring) designed and supervised intervention components. J.K., T.N., J.L., and E.L. did the data analysis. J.K., T.N., J.L., and E.L. interpreted the results and drafted the report. J.K., T.N., A.S., R.A., T.L., M.P., R.R., T.S., J.T., H.S., and M.K. obtained funding. All authors revised the article for important intellectual content.

Sponsor's Role: The funding sources had no role in the design and conduct of the study; in the collection, analysis, and interpretation of the data; or in the preparation, review, or approval of the manuscript.

\section{REFERENCES}

1. Katz S, Ford AB, Moskowitz RW, Jackson BA, Jaffe MW. Studies of illness in the aged: the index of ADL: a standardized measure of biological and psychosocial function. JAMA. 1963;185:914-919.

2. Lawton MP, Brody EM. Assessment of older people: self-maintaining and instrumental activities of daily living. Gerontologist. 1969;9(3):179-186.

3. Verbrugge LM, Jette AM. The disablement process. Soc Sci Med. 1994;38 (1):1-14.

4. Ambrose AF, Paul G, Hausdorff JM. Risk factors for falls among older adults: a review of the literature. Maturitas. 2013;75(1):51-61.

5. Hardy SE, Kang Y, Studenski SA, Degenholtz HB. Ability to walk 1/4 mile predicts subsequent disability, mortality, and health care costs. J Gen Intern Med. 2011;26(2):130-135.

6. May AM, Struijk EA, Fransen HP, et al. The impact of a healthy lifestyle on disability-adjusted life years: a prospective cohort study. BMC Med. 2015; 13:39.

7. Backholer K, Pasupathi K, Wong E, Hodge A, Stevenson C, Peeters A. The relationship between body mass index prior to old age and disability in old age. Int J Obes (Lond). 2012;36(9):1180-1186.

8. Bouillon K, Batty GD, Hamer M, et al. Cardiovascular disease risk scores in identifying future frailty: the Whitehall II prospective cohort study. Heart. 2013;99(10):737-742.

9. Houston DK, Leng X, Bray GA, et al. A long-term intensive lifestyle intervention and physical function: the look AHEAD movement and memory study. Obesity (Silver Spring). 2015;23(1):77-84.

10. Ball K, Berch DB, Helmers KF, et al. Effects of cognitive training interventions with older adults: a randomized controlled trial. JAMA. 2002;288(18): 2271-2281.

11. Ngandu T, Lehtisalo J, Levalahti E, et al. Recruitment and baseline characteristics of participants in the Finnish Geriatric Intervention Study to Prevent Cognitive Impairment and Disability (FINGER): a randomized controlled lifestyle trial. Int J Environ Res Public Health. 2014;11(9):9345-9360.

12. Kivipelto M, Solomon A, Ahtiluoto S, et al. The Finnish Geriatric Intervention Study to Prevent Cognitive Impairment and Disability (FINGER): study design and progress. Alzheimers Dement. 2013;9(6):657-665.

13. Ngandu T, Lehtisalo J, Solomon A, et al. A 2 year multidomain intervention of diet, exercise, cognitive training, and vascular risk monitoring versus control to prevent cognitive decline in at-risk elderly people (FINGER): a randomised controlled trial. Lancet. 2015;385(9984):2255-2263.

14. Strandberg TE, Levalahti E, Ngandu T, Solomon A, Kivipelto M, for the FINGER Study Group. Health-related quality of life in a multidomain intervention trial to prevent cognitive decline (FINGER). Eur Geriatr Med. 2017;8:164-167.

15. Marengoni A, Rizzuto D, Fratiglioni L, et al. The effect of a 2-year intervention consisting of diet, physical exercise, cognitive training, and monitoring 
of vascular risk on chronic morbidity: the FINGER randomized controlled trial. J Am Med Dir Assoc. 2018;19(4):355-360.e1.

16. Kivipelto M, Ngandu T, Laatikainen T, Winblad B, Soininen H, Tuomilehto J. Risk score for the prediction of dementia risk in 20 years among middle aged people: a longitudinal, population-based study. Lancet Neurol. 2006;5(9): 735-741.

17. Morris JC, Heyman A, Mohs RC, et al. The Consortium to Establish a Registry for Alzheimer's Disease (CERAD), part I: clinical and neuropsychological assessment of Alzheimer's disease. Neurology. 1989;39(9):1159-1165.

18. National Nutrition Council. Finnish Nutrition Recommendations: Diet and Physical Activity in Balance. Helsinki, Finland: Edita Publishing; 2005.

19. Nelson ME, Rejeski WJ, Blair SN, et al. Physical activity and public health in older adults: recommendation from the American College of Sports Medicine and the American Heart Association. Circulation. 2007;116(9):1094-1105.

20. Komulainen P, Kivipelto M, Lakka TA, et al. Exercise, fitness and cognitiona randomised controlled trial in older individuals: the DR's EXTRA study. Eur Geriatr Med. 2010;1:266-272.

21. Dahlin E, Neely AS, Larsson A, Backman L, Nyberg L. Transfer of learning after updating training mediated by the striatum. Science. 2008;320:1510-1512.

22. Working Group Appointed by the Finnish Medical Society Duodecim and the Finnish Hypertension Society. Hypertension: Current Care Summary. Helsinki, Finland: The Finnish Medical Society Duodecim; 2009.

23. Working Group Appointed by the Finnish Medical Society Duodecim and the Medical Advisory Board of the Finnish Diabetes Society. Diabetes: Current Care Summary. Helsinki, Finland: The Finnish Medical Society Duodecim; 2007.

24. Working Group Set Up by the Finnish Medical Society Duodecim and Finnish Society of Internal Medicine. Dyslipidaemias: Current Care Summary. Helsinki, Finland: The Finnish Medical Society Duodecim; 2009.

25. Kingston A, Collerton J, Davies K, Bond J, Robinson L, Jagger C. Losing the ability in activities of daily living in the oldest old: a hierarchic disability scale from the Newcastle 85+ study. PLoS One. 2012;7(2):e31665.

26. Guralnik JM, Simonsick EM, Ferrucci L, et al. A short physical performance battery assessing lower extremity function: association with self-reported disability and prediction of mortality and nursing home admission. J Gerontol. 1994;49(2):M85-M94.

27. Vaughan L, Leng X, La Monte MJ, Tindle HA, Cochrane BB, Shumaker SA. Functional independence in late-life: maintaining physical functioning in older adulthood predicts daily life function after age 80. J Gerontol A Biol Sci Med Sci. 2016;71((suppl 1)):S79-S86.

28. Kulmala J, Nykanen I, Hartikainen S. Frailty as a predictor of all-cause mortality in older men and women. Geriatr Gerontol Int. 2014;14(4):899-905.

29. Jacob ME, Yee LM, Diehr PH, et al. Can a healthy lifestyle compress the disabled period in older adults? J Am Geriatr Soc. 2016;64(10):1952-1961.

30. Torres JL, Lima-Costa MF, Marmot M, de Oliveira C. Wealth and disability in later life: the English Longitudinal Study of Ageing (ELSA). PLoS One. 2016;11(11):e0166825.

31. Yu R, Wong M, Chang B, et al. Trends in activities of daily living disability in a large sample of community-dwelling Chinese older adults in Hong Kong: an age-period-cohort analysis. BMJ Open. 2016;6(12):e013259.
32. Falk H, Johansson L, Ostling S, et al. Functional disability and ability 75 -year-olds: a comparison of two Swedish cohorts born 30 years apart. Age Ageing. 2014;43(5):636-641.

33. Pahor M, Guralnik JM, Ambrosius WT, et al. Effect of structured physical activity on prevention of major mobility disability in older adults: the LIFE study randomized clinical trial. JAMA. 2014;311(23):2387-2396.

34. Manini TM, Beavers DP, Pahor M, et al. Effect of physical activity on selfreported disability in older adults: results from the LIFE study. J Am Geriatr Soc. 2017;65(5):980-988.

35. Toots A, Littbrand H, Lindelof N, et al. Effects of a high-intensity functional exercise program on dependence in activities of daily living and balance in older adults with dementia. J Am Geriatr Soc. 2016;64(1): 55-64.

36. Rydwik E, Frandin K, Akner G. Effects of a physical training and nutritional intervention program in frail elderly people regarding habitual physical activity level and activities of daily living: a randomized controlled pilot study. Arch Gerontol Geriatr. 2010;51(3):283-289.

\section{SUPPORTING INFORMATION}

Additional Supporting Information may be found in the online version of this article.

Supplementary Table S1. Recorded Adherence to Intervention Components

Supplementary Table S2. Percentages of Persons With Difficulties in Daily Living Activities and Physical Performance at the Baseline and at the 12- and 24-Month Follow-Up

Supplementary Table S3. Distribution of Physical Performance Scores at Baseline and at 24 Months

Supplementary Table S4. Transitions in the Chair Rise Test According to the Baseline Score and Group, and the Difference Between the Intervention and Control Group (Percentages With 95\% Confidence Intervals)

Supplementary Table S5. Transitions in the Walking Test According to the Baseline Score and Group, and the Difference Between the Intervention and Control Group (Percentages With 95\% Confidence Intervals)

Supplementary Table S6. Transitions in the Balance Test According to the Baseline Score and Group, and the Difference Between the Intervention and Control Group (Percentages With 95\% Confidence Intervals) 